\title{
A critical evaluation of microstructure-texture-mechanical behavior heterogeneity in high pressure torsion processed CoCuFeMnNi high entropy alloy
}

\author{
Reshma Sonkusare $^{1}$, Krishanu Biswas ${ }^{1}$, Nowfal Al-Hamdany ${ }^{2}$, H. G. Brokmeier ${ }^{2}$, R. Kalsar ${ }^{3}$, \\ Norbert Schell ${ }^{4}$, N. P. Gurao ${ }^{1 *}$ \\ ${ }^{1}$ Department of Materials Science and Engineering, Indian Institute of Technology Kanpur, Kanpur-208016, India \\ ${ }^{2}$ Clausthal University of Technology (IWW-TEXMAT), Agricolastr. 6, D-38678 Clausthal-Zellerfeld, Germany \\ ${ }^{3}$ Department of Materials Engineering, Indian Institute of Science, Bangalore 560012, India \\ ${ }^{4}$ German Engineering Materials Science Center at DESY, Helmholtz-Zentrum Geesthacht, Hamburg, Germany \\ *corresponding author (email ID: npgurao@iitk.ac.in)
}

\begin{abstract}
The present study aims to understand the evolution of textural and microstructural heterogeneity and its effect on evolution of mechanical properties of an equiatomic FCC CoCuFeMnNi high entropy alloy (HEA) disc subjected to high pressure torsion (HPT). HPT was performed on disc specimen with a hydrostatic pressure of $5 \mathrm{GPa}$ for $0.1,0.5,1$ and 5 turns at room temperature where the hardness saturated at $1941 \mathrm{MPa}$ at the periphery of the sample after five turns. Synchrotron diffraction texture analysis of five-turn HPT sample reveals characteristic shear

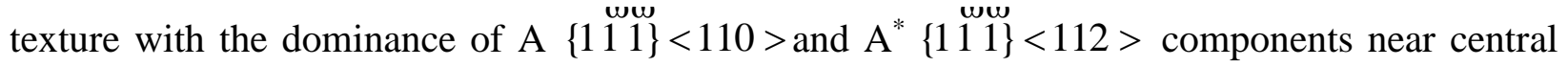
region of the disc and it shifts to $C\{001\}<110>$ component near the periphery of the disc. X-ray
\end{abstract}


diffraction analysis shows decrease in crystalline size with simultaneous increase in dislocation density for five-turn HPT sample with increasing strain from centre to the periphery of the disc. Microstructural analysis using electron back scatter diffraction and transmission electron microscopy indicates extensive grain fragmentation $\approx \approx 55 \mathrm{~nm}$ at the periphery of five-turn sample). The evolution of hardness from centre to the periphery of the disc cannot be explained only on the basis of evolution of grain size and dislocation density. The increase in contribution from solid solution strengthening due to partial dissolution of copper rich nano-clusters is expected to be the underlying cause for increase in the hardness. Thus, evolution of gradient microstructure, texture, and chemistry opens up new vistas for designing functionally graded materials for engineering materials.

Keywords: High Entropy Alloy; High Pressure Torsion; Shear Texture; Synchrotron Diffraction; Heterogeneity; Strengthening Mechanisms 


\section{Introduction}

High pressure torsion (HPT) is a severe plastic deformation (SPD) technique in which high hydrostatic pressure is applied on a disc sample along with torsional straining to obtain truly bulk nanostructured materials (average grain size $<100 \mathrm{~nm}$ ) with highest fraction of high angle grain boundaries $[1,2,3,4]$. All SPD processes are inherently heterogeneous in nature, especially the HPT process which is primarily shear based [5,6] leading to shear strain-induced grain refinement in the material $[7,8]$. This technique is capable of imposing unlimited magnitude of strain in a single operation, due to the high hydrostatic pressure. Since shear strain during this technique varies as a function of the distance from the centre of the disc, there is evolution of heterogeneous gradient microstructure [9]. The systematic change in the microstructure on a macroscopic scale leads to synergetic strengthening that can be explored to achieve excellent strength and ductility [10]. The fine grain structure at the periphery imparts strength and the gradually increasing grain size towards the centre provides ductility to the material. The microstructure in HPT processed materials has a gradient in grain size, shear strain, hardness and texture. These effects are further compounded in high entropy alloys (HEAs) which are complex concentrated solid solution alloys $[11,12,13,14,15]$. Conventional alloys are based on one principal element, but HEAs contain minimum five elements in equimolar or near equimolar proportions, which makes them compositionally complex [16, 17, 18, 19, 20, 21]. Deformation processes like work hardening and grain refinement are strongly related to its crystallographic texture $[22,23]$. However, study of texture evolution in HPT processed high entropy alloys has not been reported till date. 
Of particular importance is the role of geometrically necessary dislocations (GNDs) in shear process that play an important role in the grain fragmentation leading to a reduction in grain size by three orders of magnitude from tens of micron to tens of nanometer in HPT [24, 25]. The presence of higher fraction of GNDs plays an important role in the onset of continuous dynamic recrystallization which contributes to the overall grain refinement process. The texture evolution in torsion is characterized by rigid body rotation as well as plastic spin. In torsion, texture stability requires the counterbalance presence of high glide rotation rates (or plastic spin rates), due to presence of high rigid body rotation rates [26]. Toth et al. [27] have discussed the orientation persistence parameter S given by

$S=\ln \left(\frac{\dot{\bar{\epsilon}}}{|\dot{\Omega}|}\right)$

where, $\dot{\bar{\epsilon}}=\dot{\gamma} / 3$ and $|\dot{\Omega}|=$ magnitude of lattice rotation rate vector. When the lattice spin is low, orientation can persist over a large strain. High value of orientation persistence indicates relative orientation stability i.e. orientation remain strong over a large range of strain. Thus, the evolution of microstructure in terms of dislocation sub-structure contributing to grain refinement and micro-texture evolution in HPT still remains an open issue for conventional metals and alloys as well as complex concentrated solid solutions like HEAs. The large strain deformation of HEAs is important to study the effect of compositional complexity on phase stability and evolution of microstructure, texture and hardness in HEAs.

One such interesting FCC HEA developed by the present authors is characterized by low stacking fault energy and presence of copper rich nano-clusters of size $\sim 2.5 \mathrm{~nm}$ with hardness of $539 \mathrm{MPa}$ in the fully annealed condition [28, 29, 30, 31]. It is to be mentioned here that copper 
has positive enthalpy of mixing with remaining elements in the HEA and there is separation of a copper rich phase at high temperature and hence it is worthwhile to study phase stability under high strain energy regime. In order to address this issue, a complete investigation for heterogeneity in mechanical properties, microstructure and texture from the centre to the periphery of the five-turn HPT processed disc of FCC CoCuFeMnNi HEA was studied. To this end, micro-focus synchrotron diffraction, electron backscatter diffraction (EBSD), nanoindentation and viscoplastic self-consistent (VPSC) simulations were employed to unravel the deformation mechanisms. Texture evolution was studied using synchrotron radiation, from the centre to the periphery of five-turn sample. Although the major focus of the manuscript is to study the evolution of heterogeneity in microstructure, texture and mechanical behavior in fiveturn HPT sample; microstructural evolution for $0.1,0.5$ and 1 turn HPT samples has been reported in details. As the evolution of texture and microstructure is a sequential process, analysis of samples deformed to lower strain is expected to provide better insight into the operative micro-mechanisms. For clarity, EBSD data has been extensively used to explain the evolution of microstructure and its heterogeneity from initial to final stages of HPT. Contribution from various strengthening mechanisms was calculated to explain the heterogeneity in mechanical behavior and establish the microstructure-texture-mechanical behaviour paradigm for HPT processed CoCuFeMnNi HEA.

\section{Materials and method}

\subsection{Processing}

An alloy button of equiatomic CoCuFeMnNi HEA was melted in a vacuum arc melting furnace, using non-consumable tungsten electrode and water cooled copper hearth, in the presence of 
argon atmosphere. The button was sealed in a quartz tube and then annealed at $1273 \mathrm{~K}$ for 10 hour, followed by water quenching. Small disc specimens were machined from the button using electric discharge machining (EDM), with the dimension of $8 \mathrm{~mm}$ diameter and $0.8 \mathrm{~mm}$ thickness. The disc were processed via high pressure torsion, with hydrostatic pressure of $5 \mathrm{GPa}$. The von Mises strain $\left(\varepsilon_{v m}\right)$ developed during HPT can be calculated using the following equation.

$\varepsilon_{v m}=\frac{2 \pi r N}{\sqrt{3} h}$

Here, $\mathrm{r}=$ radius from the centre of the disc, $\mathrm{N}=$ number of turns, $\mathrm{h}=$ average thickness of the disc. Revolutions of 0.1, 0.5, 1 and 5 turns were imposed on the discs.

\subsection{Characterization}

Macro-texture measurements were conducted at different radius of five-turn sample, using Synchrotron source (DESY Photon Science, Hamburg, Germany), with a wavelength of 0.0142 $\mathrm{nm}$, in transmission mode. The beam energy, spot size and sample to detector distance was 87 $\mathrm{keV}, 300 \mu \mathrm{m} \times 300 \mu \mathrm{m}$ and $1000 \mathrm{~mm}$, respectively. A solid state area detector (Perkin Elmer) was used to acquire the X-ray diffraction pattern. The instrument was first calibrated with standard sample $\left(\mathrm{LaB}_{6}\right)$ and then measurements were carried out for five-turn HPT sample with exposure time of 1 second per image. The diffraction patterns were analyzed using FIT2D software and pole figures and orientation distribution function (ODF) were generated using CUBEODF software. The ODF was determined from incomplete pole figures obtained from synchrotron diffraction using monoclinic sample symmetry and recalculated (111), (200) and (220) complete pole figures were determined. Single shot scan, obtained using synchrotron, was 
utilized for determination of crystallite size, micro-strain and dislocation density as a function of radius in five-turn sample and analysis was carried out in commercially available Xpert Highscore plus PANalytical software, based on Williamson-Hall equation. First three peaks of the XRD pattern were selected to fit Williamson-Hall equation. The schematic of synchrotron is shown in Figure 1(a) and the location at which various characterization has been done is shown in Figure 1(b).

Micro-texture measurements were carried out using electron backscatter diffraction (EBSD) in a JEOL JSM-7100F Field Emission Scanning Electron Microscope equipped with Oxford EBSD detector. The samples were prepared by polishing with emery paper followed by alumina cloth polishing and colloidal silica polishing. Step size of $0.15 \mu \mathrm{m}$ and binning mode of $2 \times 2$ was used to scan the sample. The analysis was carried out using TSL-OIM software version 7.2. The orientation distribution function (ODF) was calculated from EBSD data using monoclinic symmetry of HPT samples.

Transmission Electron Microscopy (TEM) was carried out on a $3 \mathrm{~mm}$ disc cut from the periphery of 0.1-turn, one-turn and five-turn HPT sample. The disc was ground using emery paper upto $80 \mu \mathrm{m}$, followed by dimpling grinder (Gatan, model 656, USA). It was then subjected to precision ion polishing system (Gatan, model 691, USA). Argon gas ions were purged to create perforation at the centre of the disc. The sample was then observed under JEOL 2000 FXII TEM. To resolve nano-sized grains at the periphery of five-turn sample, TEM-OIM was carried out. For TEM-OIM, FEI Technai F-30 transmission electron microscope (TEM), operating at $300 \mathrm{kV}$ voltage, was used. Orientation imaging was performed using precession electron diffraction (PED) in the TEM-based orientation imaging microscopic analysis with 
NanoMegas (Digital/ASTAR) which has resolution of the order of a nanometer. The $3 \mathrm{~mm}$ disc sample prepared for TEM was utilized for TEM-OIM also.

Vickers micro-indentation was carried out on 0.1 turn, one-turn and five-turn sample from centre to periphery at five different angles $\left(0^{\circ}, 22.5^{\circ}, 45^{\circ}, 67.5^{\circ}\right.$ and $\left.90^{\circ}\right)$. A load of $500 \mathrm{~g}$ was applied on the sample for a dwell time of $10 \mathrm{~s}$. The data obtained was plotted as 2D contour maps using OriginPro 8.5 software. Instrumented nano-indentation (Hysitron, model TI750) with Berkovich indenter [32] was carried out on five-turn HPT sample at different radius from the centre. Maximum load of $1000 \mu \mathrm{N}$ was used to obtain load vs depth curve, hardness value and elastic modulus at various radius.

\subsection{Simulations}

Viscoplastic self-consistent (VPSC) simulation was utilized to simulate the HPT texture for different values of shear strain in order to understand deformation micro-mechanisms. In VPSC, a grain in a polycrystalline material is considered as an inclusion in a homogeneous equivalent medium (HEM) having the average property of the polycrystal $[33,34]$. The strain rate within the grain is given by the following equation.

$D_{i j}^{c}=\gamma^{0} \sum_{s} m_{i j}^{s}\left(\frac{m^{s}: \sigma}{\tau^{s}}\right)^{n}=\gamma^{0} \sum_{s} m_{i j}^{s} m_{k l}^{s}\left(\frac{m^{s}: \sigma}{\tau^{s}}\right)^{n-1}$

Here, $\mathrm{s}=$ slip system, $\gamma^{0}=$ normalizing strain rate, $m^{s}=$ geometric Schmid tensor, $\tau^{s}=$ threshold value of stress and $n=$ rate sensitivity inverse. The evolution of critical resolved shear stress (CRSS) for slip or twinning with respect to strain is governed by the Voce law [35] as given below. 
$\tau(\Gamma)=\tau_{0}+\left(\tau_{1}+\theta_{1} \Gamma\right)\left[1-\exp \left(\frac{-\theta_{0} \Gamma}{\tau_{1}}\right)\right]$

The four parameters $\tau_{0}, \tau_{1}, \theta_{0}$ and $\theta_{1}$ contribute to hardening of slip systems. In the present work, 2000 grains with random texture was used as input file and VPSC-7 with affine approach was used to model shear texture during HPT for two cases (a) considering octahedral as well as partial slip systems and (b) considering only octahedral slip systems. The simulations were carried out only till strain of three to qualitatively show the evolution of texture as the code cannot handle large shear strain. Though the actual shear strain in five-turn HPT sample is 157, the present simulation is an attempt to show the formation of characteristic shear texture in HEA with and without the operation of partial slip.

\section{Results}

\subsection{Evolution of texture}

Textural evolution provides important information for understanding the deformation behavior during HPT processing. The torsion texture can be described as $\{h k l\}\langle u v w\rangle$, where $\{h k l\}$ is parallel to shear plane and $\langle u v w\rangle$ is parallel to shear direction. Figure 2(a) shows the ideal shear texture components $\left(A, \bar{A}, A_{1}^{*}, A_{2}^{*}, B, \bar{B}, C\right)$ in (111) pole figure. The Euler angles and $\{h k l\}<u v w>$ for the main FCC shear texture components is shown in Table I and it is discussed in details by Li et al. [36] and Toth et al. [20]. Figure 2(b) shows the (111), (200) and (220) pole figures from the centre to the periphery of the five-turn HPT sample. It can be observed that the texture is heterogeneous as a function of radius and there is evolution of characteristic shear texture as observed in FCC metals and alloys. However, the texture obtained is extremely weak due to grain fragmentation at high strain. The texture at the periphery (Z1) does not resemble 
with shear texture because there was lateral flow of material apart from shear strain. There is a continuous strengthening of components $\mathrm{B}$ and $\mathrm{C}$ and continuous weakening of $\mathrm{A}$ and $\mathrm{A}^{*}$ from the centre to periphery.

\subsection{Evolution of microstructure and micro-texture}

In order to understand the microstructural evolution depicting the heterogeneity from centre to periphery, EBSD analysis was carried out from different sections of 0.1-turn sample subjected to different amount of shear strain. A schematic of various sections analyzed is shown in Figure 1(b). Figure 3 shows the crystal orientation or inverse pole figure (IPF) maps of the annealed sample and HPT samples at different locations. EBSD scans were performed at the central region of $0.1,0.5,1$, and 5 turn HPT samples as well as at the periphery and middle region of 0.1-turn HPT sample to study the evolution of microstructure as a function of different number of turns and the heterogeneity of microstructural evolution in 0.1-turn sample respectively. The annealed sample has coarse equiaxed grains of $37 \mu \mathrm{m}$ average grain size and is characterized by the presence of annealing twins typical of medium SFE FCC materials like copper. The colour gradients seen in IPF maps of HPT samples indicate orientation gradient due to dislocation activity. The grains are deformed even at the centre of the sample, whereas the grains at the periphery are elongated. It can be observed that the average grain size decreases as the shear strain increases with number of turns as well as from centre to periphery for 0.1-turn sample. Table II also indicates that GND density increases as the distance from centre to periphery increases for 0.1-turn sample as well as with increase in number of turns at the centre of HPT discs. Due to the resolution limit of EBSD technique, it was not possible to obtain data at the periphery of $0.5,1$, and 5 turn samples deformed to higher strain levels. 
Intragranular misorientation determined in terms of kernel average misorientation (KAM) increases from the centre of the disc to the periphery and is characterized by shifting of peak KAM to higher value. This is a clear indication of increase in misorientation within the grains that leads to grain fragmentation in 0.1-turn HPT sample (Figure 4(a)). KAM distribution at the centre of the disc also shifts to right with increase in number of HPT turns (Figure 4(b)) indicating higher intragranular misorientation with increase in number of turns of HPT. Information about various type of grain boundaries and intragranular misorientation in terms of grain reference orientation deviation (GROD) as well as geometrically necessary dislocation density was also determined from EBSD data and is displayed in Table II. Gradual increase in GROD values and fraction of high angle grain boundaries is observed with increase in strain. Figure 5(a) shows the grain boundary map at the periphery of five-turn HPT sample obtained from TEM-OIM. The black lines represent high angle grain boundaries $\left(\theta \geq 15^{\circ}\right)$ and red lines represent low angle grain boundaries $\left(\theta=2-15^{\circ}\right)$. Severe grain fragmentation can be observed, with average grain size of $55.4 \pm 33.4 \mathrm{~nm}$. Taylor factor map at the periphery of 5 turn sample is shown in Figure 5(b). The grains are equiaxed even at the shear strain of 157 and the grain fragmentation is uniform indicating Taylor kind of deformation of the nano-grains. Figure 6 shows the bright field TEM images at the periphery of 0.1-turn, one-turn and five-turn sample. Evolution of distinct dislocation structure is observed at the periphery of the one-turn HPT sample in Figure 6(a) while Figure 6(b) shows extensive grain refinement and corresponding SAD pattern shows a spotty ring indicating nanosize grains.

\subsection{Evolution of hardness}

We have measured micro-hardness of all the HPT processed samples. However, we shall present the salient results to bring about the details of deformation behaviour of the HEA at different 
strain. Figure 7 shows the hardness evolution from centre to periphery in 0.1-turn, one-turn and five-turn sample using 2D contour maps. The hardness increases as a function of radius and reaches saturation at $1941 \mathrm{MPa}$ (supplementary data S.III) which is more than 3.5 times the hardness of the homogenized sample. The saturation hardness is achieved in five-turn sample itself and even ten-turn HPT sample showed the same value of hardness at the periphery of the HPT processed disc.

Figure 8(a) shows the load (P) vs depth (d) curve from nano-indentation for different radius of five-turn sample. The P-d curves shift to the left (lower indentation depth) indicating rise in hardness from centre to periphery. Figure 8(b) shows the variation of hardness and elastic modulus as a function of distance from the centre of the disc. The hardness increases from centre to periphery whereas, elastic modulus remains almost constant from centre to periphery of the HPT disc. This unique trend in hardness is attributed to partial dissolution of copper rich nanoclusters during high pressure torsion which leads to increase in solid solution strengthening and hence increases the hardness. Atom probe tomography of five turn HPT sample (supplementary data Figure S2) indicated reduction in cluster size from 2.5 to $1.5 \mathrm{~nm}$ with corresponding increase in copper contents from 8 to 17 atomic percentage in the matrix (supplementary data Table S1) [37,38,39]. The hardness obtained using nano-indentation is always higher than that obtained using micro-indentation due to higher contribution of GNDs for smaller indents [40,41].

\section{Discussion}

A thorough analysis of the evolution of microstructure, texture and mechanical behaviour in terms of unique evolution of hardness, clearly indicates a combinatorial effect of multiple micro- 
mechanisms operative during high pressure torsion. The role of processes like grain fragmentation, evolution and stability of texture components and evolution of mechanical properties relevant for HPT will be discussed for the special case of CoCuFeMnNi HEA.

\subsection{Evolution of texture and microstructure}

X-ray diffraction (using synchrotron) was carried out to determine the average crystallite size and micro-strain in five-turn sample using Williamson-Hall method [42].

$B \cos \theta_{B}=\frac{K \lambda}{D}+4 \varepsilon \sin \theta_{B}$

Here, $B$ is the peak broadening (FWHM, $\left.=\sqrt{B_{\text {sample }}^{2}-B_{\text {instrument }}^{2}}\right), \theta_{B}$ is the Bragg angle, $K$ is constant, $\lambda$ is the wavelength, $D$ is crystallite size and $\varepsilon$ is micro-strain. The dislocation density ( $\rho$ ) was calculated using the following formula [43].

$\rho=\frac{2 \sqrt{3}\langle\varepsilon\rangle}{D b}$

Here, $\varepsilon$ is the micro-strain, $D$ is the crystallite size and $b$ is the Burgers vector. The crystallite size decreases (44 to $26 \mathrm{~nm}$ ) from the centre to the periphery of five-turn sample while the micro-strain and dislocation density increases as a function of radius (Figure 8(c)). TEM-OIM at the periphery of five-turn sample showed number average grain size of $25 \mathrm{~nm}$ which is in good agreement with the X-ray diffraction results while the area average grain size was $55 \mathrm{~nm}$. The high dislocation density can be attributed to reduced annihilation rate of dislocations during deformation because of high Peierls-Nabarro stress to move the dislocations and restricted cross slip due to low stacking fault energy in the concentrated HEA. 
The trend for crystallite size and dislocation density shown in Figure 8(c) is characterized by sudden decrease from the centre followed with gradual decrease from centre to periphery of the HPT disc. This implies that the grain fragmentation rate and evolution of dislocation density is faster near the centre that is at low effective strain while it becomes sluggish at the periphery (high strain). Chen et al. [11] have described the effect of initial grain size on the plastic deformation and grain fragmentation mechanism during SPD processes. It was observed that grain refinement is dominated by deformation twinning in coarse grain sample while dislocation slip is prevalent in fine grained sample. Moreover, it was observed that the grain refinement rates are high for coarse grains and low for fine grains.

It has been reported by Naghdy et al. [44] that for high SFE materials, a copper-type texture with dominance of $\mathrm{C}$ component $\{001\}<110>$ and for low SFE materials, a brass-type texture with dominance of $\mathrm{B}$ component $(\stackrel{\omega}{1} 12\}<110>$ is observed. In the present work, both $\mathrm{B}$ and $\mathrm{C}$ components increases from centre to periphery, indicating medium SFE characteristics. Beladi et al. [45] reported that dynamic recrystallization (DRX) grains have dominance of A component during torsion. Since A component is decreasing from centre to periphery of five-turn sample, the tendency of DRX is also decreasing. In the present alloy, grain refinement process occurs by the process of continuous dynamic recrystallization (cDRX) that involves short range grain boundary migration in low SFE FCC materials to form new sub-micron and nano-crystalline grains [46]. Sub-grain or cell structure observed in TEM micrograph of one-turn sample is an indication of cDRX process where LAGBs progressively evolve into HAGBs at larger deformations and there is no clear nucleation and growth stage. The accumulation of dislocations near the LAGBs increases with deformation which increase the misorientation and eventually when it reaches a critical misorientation $\left(\theta_{c} \approx 15^{\circ}\right)$, HAGBs are formed. The recrystallization 
occurs uniformly and new grains forms by progressive rotation of sub-grains and microstructure evolution exhibit a continuous character.

During HPT, the grains get sheared and become elliptical in shape and the aspect ratio of the grains should increase with shear strain as discussed by Canova et al. [17]. Grain refinement during HPT is also aided by deformation twins and shear banding [20]. Since HPT samples experience inhomogeneous straining, different regions of a grain experience different straining and therefore bands are formed, separated by geometrically necessary boundaries (GNBs). Apart from GNBs, incidental dislocation boundaries (IDBs) can also form by statistical trapping of dislocations [47]. As the strain increases during HPT, misorientation of GNBs and IDBs also increases, leading to formation of HAGBs and therefore grain refinement occurs. In the present case, cDRX has reached steady state that leads to a saturation hardness value. However, there is still heterogeneity in hardness value indicating the heterogeneity of microstructure and texture in five-turn HPT sample.

\subsection{Viscoplastic self consistent simulations}

The VPSC simulation was carried out to obtain evolution of texture in the HPT sample. Twelve octahedral slip system $\{111\}<1 \overline{1} 0>$ and twelve partial slip system $\{111\}<11 \overline{2}>$ were considered as the operating mode of deformation of FCC CoCuFeMnNi HEA [27] during the simulations in first case and only octahedral slip system was considered for the other. The Voce hardening parameters used are shown in Table III. Twins observed at the centre of half-turn sample in EBSD [12] justifies considering partial slip system along with octahedral slip system. The initial texture with random 2000 orientations was used and simulation was carried out with Affine 
approximation using an inverse strain rate sensitivity exponent of 20 . The following velocity gradient was employed for the simulation.

$$
L=\left(\begin{array}{lll}
0 & \gamma & 0 \\
0 & 0 & 0 \\
0 & 0 & 0
\end{array}\right)
$$

The texture obtained for the two conditions were plotted using Resmat software. The simulated 111 pole figure with octahedral + partial slip system (Figure 9(a)) shows a reasonable match with the experimental results compared to pole figure simulated considering only octahedral slip system (Figure 9(b)). The experimental texture at the periphery of five-turn HPT sample is deformed to a shear strain of 157 so it is not prudent to compare the simulation results on a one to one basis. Nevertheless, it is clear that characteristic shear texture can be produced by the operation of partial planar slip and octahedral slip in the HEA. The observation of weaker texture even at much higher strain is attributed to grain fragmentation [48] and occurrence of cDRX which are not incorporated in the simulations.

\subsection{Taylor factor and grain fragmentation}

Taylor factor is related to the crystallographic orientation and is defined as total slip amplitude corresponding to a unit macroscopic strain increase [49,50]. For plastically isotropic materials, Taylor factor is given by the following equation [51]

$$
M=\frac{Y}{\tau_{0}}=\frac{d \Gamma}{d \varepsilon}=\frac{\sum \Delta \gamma}{\Delta \varepsilon}
$$

Here, $\mathrm{Y}=$ yield strength, $\tau_{0}=$ CRSS of a reference single crystal, $d \Gamma=$ algebraic sum of individual shear contributions of slip systems at grain level, $d \varepsilon=\Delta \varepsilon=$ incremental macroscopic 
strain. Grain with highest Taylor factor will experience highest microscopic slip, for a given value of $\Delta \varepsilon$. The grain orientation with high Taylor factor has higher dislocation density and greater misorientation angles and can be expected to get consumed during dynamic recrystallization process. Jonas and Toth [52,53] have calculated the Taylor factor for different stable end orientations and Taylor factor increases in the order $\mathrm{A}<\mathrm{A} *<\mathrm{B}<\mathrm{C}$. It is expected that grains with $\mathrm{C}$ component will have the highest dislocation density than grains with $\mathrm{A}$ component [54].

The microstructure evolution in 0.1-turn HPT sample (Figure 3 and Table II) shows increase in GND density with increase in strain from centre to periphery. This implies that the deformation is heterogeneous in nature and it increases from centre to periphery. The grains become elongated as strain increases during the HPT process. GND density depends on lattice curvature, which is calculated from measuring the orientations within small neighbouring material volumes. After that, the dislocation density tensor is used to derive the GND density [55]. The deformation related to HPT is given by distortion tensor, $\beta$ [56].

$$
\beta=\left(\begin{array}{ccc}
e / 2 & \gamma & 0 \\
0 & -e & 0 \\
0 & 0 & e / 2
\end{array}\right)
$$

Where, $\mathrm{e}=$ flow of excess material at lower deformation and $\gamma=$ shear deformation. Torsion can be accommodated by two slip systems unlike tension or compression where five independent slip systems are required. Moreover, there is rigid body rotation during torsion and is absent in tension or compression. It has been reported by Hughes et al. [57] that higher amount of A component indicate uniform distribution of slip via extensive cross slip and hence low Taylor factor components are stabilized. C component has high Taylor factor and grains reorient themselves and undergo grain fragmentation and this occurs in low SFE materials (supplementary data S.IV). Grains with high Taylor factor experience more dislocation activity 
per unit strain and grains with low Taylor factor has less dislocation activity per unit strain to accommodate externally imposed strain and hence deform plastically without significant reorientation. In the present case, A component decreases from centre to periphery indicating uniform slip distribution at the centre and grain reorientation and fragmentation at the periphery which is evident from the microstructure as well. Less microscopic slip activity at the centre implies lower GND density and hence lower intragranular misorientation. Weakening of the texture at high strain or high strain rate occurs due to grain fragmentation.

\subsection{Evolution of strength}

The strength contribution from various strengthening mechanisms [58,59,60] like frictional stress, strain/Taylor hardening [61,62], Hall-Petch hardening [63,64], cluster strengthening [65] and solid solution strengthening [59] were calculated for Z1, Z2, Z3, and Z4 positions, as shown in Table IV. Here, we have assumed a linear superposition of all five mechanisms because the interaction between the above mechanisms in high entropy alloys is not known yet and hence their independent contribution is considered. No attempt has been made to calculate strengthening contribution at the centre of the disc as the microstructure is very heterogeneous. The yield strength increases from $1555 \mathrm{MPa}$ at $1 \mathrm{~mm}$ from the centre to $1941 \mathrm{MPa}$ at the periphery of the five-turn HPT disc. Yield strength was calculated from the micro-hardness using the equation $\sigma_{Y S}=H / 3$ [58]. The frictional stress $\left(\sigma_{F R}\right)$, calculated using rule of mixtures [58], was found to remain constant as the elements and their proportion is not changing. Strain hardening was calculated using the formula $\sigma_{S H}=M \alpha G b \sqrt{\rho}$, where $M$ is the Taylor factor, $\alpha$ is proportionality constant (depends on the nature of dislocation [61], 0.16 in present case), $G$ is the shear modulus (rule of mixture), $b$ is Burgers vector (determined from synchrotron data) and $\rho$ is dislocation density (determined from synchrotron data). It was found that the strain hardening 
increases slightly from 334 to $354 \mathrm{MPa}$ due slight increase in dislocation density. The grain size determined from synchrotron data, was found to decrease from the centre to the periphery and hence led to Hall-Petch hardening from 865 to $914 \mathrm{MPa}$. Hall Petch hardening was calculated using the formula $\sigma_{H P}=k_{H P} / \sqrt{d}$, where $k_{H P}$ is the Hall Petch coefficient and $d$ is the grain size. The $k_{H P}$ of HPT processed Cantor's alloy (CoCrFeMnNi HEA) was used in the present case [57]. The equation for determining contribution of solid solution strengthening in complex concentrated alloys is not available in the literature yet and cluster strengthening cannot be calculated without knowing the fraction of copper rich nano-clusters. Therefore, the combined contribution of solid solution and cluster strengthening was calculated by subtracting the contribution of other 3 mechanisms $\left(\sigma_{F R}, \sigma_{S H}, \sigma_{H P}\right)$ from the total yield strength. It was found that the contribution of solid solution $\left(\sigma_{S S}\right)$ and cluster strengthening $\left(\sigma_{C L}\right)$ is tremendous and it increases from 322 to $639 \mathrm{MPa}$. As the distance from centre increases, shear strain increases and it may increase the dissolution of copper nano-clusters $[28,29,66,67,68]$, increasing the copper concentration in the matrix and therefore leads to increase in solid solution strengthening.

Figure 10(a) shows the schematic of hardness and microstructure evolution in different HPT samples. Edalati et al. [69] found that the temperature rises during HPT are relatively minor when compared with the melting temperatures of the processed metals. It was concluded that the microstructural evolutions occurring during HPT processing are not significantly influenced by the temperature rise. On the contrary, the formation of high-angle grain boundaries through dynamic recrystallization (DRX) is due to the presence of large volumes of lattice defects. Figure 10(b) shows the contribution of various strengthening mechanisms operative during HPT process, from the centre to the periphery of five-turn sample. The success of phenomenological equations of different strengthening mechanisms to explain the experimental hardness evolution 
in five-turn HPT sample also indicates high fidelity of constants like shear modulus and HallPetch co-efficient used in different phenomenological approaches for the CoCuFeMnNi HEA. Thus it is evident that the evolution of heterogeneous microstructure in terms of grain size, dislocation density and texture leads to evolution of heterogeneous mechanical properties like hardness and elastic modulus in CoCuFeMnNi HEA during high pressure torsion.

\section{Summary and conclusions}

The heterogeneity in microstructure, texture and mechanical behavior of five-turn HPT processed single phase $\mathrm{CoCuFeMnNi}$ high entropy alloy has been studied in the present investigation. Based on the experimental data and simulations that were carried out, following conclusions can be drawn.

1) The textural investigation indicates the development of typical FCC shear texture with the dominance of $A\{111\}\}<110>$ and $A^{*}\{111\}<<112>$ components at initial stages of deformation and it shifts to $\mathrm{C}\{001\}<110>$ component at later stages of deformation. The observation of characteristic shear texture can be explained on the basis of operation of octahedral and partial slip while grain fragmentation leads to weaker texture at higher strain.

2) Microstructural investigation indicates heterogeneous microstructure and leads to strong grain size refinement from $37 \mu \mathrm{m}$ to $55 \mathrm{~nm}$ at a shear strain of 157 in five-turn HPT sample. This is caused because of deformation twinning and deformation banding in addition to octahedral and partial slip. The grain refinement process is dominant at the periphery and reaches saturation in hardness after five turns of HPT. 
3) Strain hardening and dislocation hardening leads to increase in hardness (or yield strength) from centre to periphery of five turn HPT sample and it is expected that the contribution of solid solution and cluster strengthening increases from centre to periphery.

4) The heterogeneity of nano-chemistry played an important role in the deformation of $\mathrm{CoCuFeMnNi} \mathrm{HEA} \mathrm{apart} \mathrm{from} \mathrm{heterogeneity} \mathrm{of} \mathrm{mechanical} \mathrm{property,} \mathrm{microstructure} \mathrm{and}$ texture. It can be anticipated that the heterogeneity of grain size, chemistry and microstructure can lead to improved mechanical properties. The potential of generating gradient microstructure, texture, and chemistry opens up a new avenue to generate functionally graded materials for engineering materials.

\section{Acknowledgements}

The authors would like to thank the DST-DESY program for providing opportunity and financial assistance for carrying out synchrotron experiments in Germany. The authors acknowledge the facilities at Texture Laboratory, Advanced Centre for Materials Science, IIT Kanpur. The authors also want to acknowledge Dr. Sanjit Bhowmick and Dr. Manimunda Praveena from Bruker Nano Surfaces, Minneapolis, USA for carrying out nano-indentation of five-turn HPT sample.

\section{References}

[1] Reshma Sonkusare, Nimish Khandelwal, Pradipta Ghosh, Krishanu Biswas, N. P. Gurao. A comparative study on the evolution of microstructure and hardness during monotonic and cyclic high pressure torsion of $\mathrm{CoCuFeMnNi}$ high entropy alloy. Journal of Materials Research 34 (2019) 732-743. DOI: https://doi.org/10.1557/jmr.2018.479

[2] A. P. Zhilyaev, G. V. Nurislamova, B.-K. Kim, M. D. Baro', J. A. Szpunar, T. G. Langdon. Experimental parameters influencing grain refinement and microstructural evolution during high-pressure torsion. Acta Materialia 51 (2003) 753-765. DOI: https://doi.org/10.1016/S1359$\underline{6454(02) 00466-4}$ 
[3] A. Vorhauer and R. Pippan. On the homogeneity of deformation by high pressure torsion. Scripta Materialia 51 (2004) 921-925. DOI: https://doi.org/10.1016/j.scriptamat.2004.04.025

[4] Alexander P. Zhilyaev, Terence G. Langdon. Using high-pressure torsion for metal processing: Fundamentals and applications. Progress in Materials Science 53 (2008) 893-979. DOI: https://doi.org/10.1016/j.pmatsci.2008.03.002

[5] Subrata Panda, Laszlo S. Toth, Jean-Jacques Fundenberger, Olivier Perroud, Julien Guyon, Jianxin Zou, Thierry Grosdidier. Analysis of heterogeneities in strain and microstructure in aluminium alloy and magnesium processed by high-pressure torsion. Materials Characterization 123 (2017) 159-165. DOI: http://dx.doi.org/10.1016\%2Fj.matchar.2016.11.027

[6] Mohammad Jahedi, Irene J. Beyerlein, Mohammad Hossein Paydar, Shijian Zheng, Ting Xiong, Marko Knezevic. Effects of Pressure and Number of Turns on Microstructural Homogeneity Developed in High-Pressure Double Torsion. Metallurgical and Materials Transactions A 48 (2017) 1249-1263. DOI: https://doi.org/10.1007/s11661-016-3923-7

[7] Kaveh Edalati and Zenji Horita. A review on high-pressure torsion (HPT) from 1935 to 1988. Materials Science \& Engineering A 652 (2016) 325-352. DOI: https://doi.org/10.1016/j.msea.2015.11.074

[8] Y. Estrin and A. Vinogradov. Extreme grain refinement by severe plastic deformation: A wealth of challenging science. Acta Materialia 61 (2013) 782-817. DOI: https://doi.org/10.1016/j.actamat.2012.10.038

[9] Jordan Moering, Xiaolong Ma, Guizhen Chen, Pifeng Miao, Guozhong Li, Gang Qian, Suveen Mathaudhu, Yuntian Zhu. The role of shear strain on texture and microstructural gradients in low carbon steel processed by Surface Mechanical Attrition Treatment. Scripta Materialia 108 (2015) 100-103. DOI: http://dx.doi.org/10.1016/j.scriptamat.2015.06.027

[10] X. L. Wu, P. Jiang, L. Chen, J. F. Zhang, F. P. Yuan, Y. T. Zhu. Synergetic Strengthening by Gradient Structure. Materials Research Letters 2 (2014) 185-191. DOI: https://doi.org/10.1080/21663831.2014.935821

[11] W Skrotzki, A Pukenas, B Joni, E Odor, T Ungar, A Hohenwarter, R Pippan, E P George. Microstructure and texture evolution during severe plastic deformation of CrMnFeCoNi highentropy alloy. IOP Conference Series: Materials Science and Engineering 194 (2017) 012028. DOI: $10.1088 / 1757-899 X / 194 / 1 / 012028$

[12] Ruixiao Zheng, Jing Chen, Wenlong Xiao, Chaoli Ma. Microstructure and tensile properties of nanocrystalline $(\mathrm{FeNiCoCu})_{1-x} \mathrm{Ti}_{\mathrm{x}} \mathrm{Al}_{\mathrm{x}}$ high entropy alloys processed by high pressure torsion. Intermetallics 74 (2016) 38-45. DOI: http://dx.doi.org/10.1016/j.intermet.2016.05.008

[13] Amit S. Sharma, Surekha Yadav, Krishanu Biswas, Bikramjit Basu. High-entropy alloys and metallic nanocomposites: Processing challenges, microstructure development and property enhancement. Materials Science \& Engineering R 131 (2018) 1-42. DOI: $\underline{10.1016 / \text { j.mser.2018.04.003 }}$

[14] A. Patel, I. Wani, S. R. Reddy, S. Narayanaswamy, A. Lozinko, R. Saha, S. Guo, P. P. Bhattacharjee. Strain-path controlled microstructure, texture and hardness evolution in cryodeformed $\mathrm{AlCoCrFeNi}$.1 eutectic high entropy alloy. Intermetallics 97 (2018) 12-21. DOI: https://doi.org/10.1016/j.intermet.2018.03.007 
[15] J. Moon, M. J. Jang, J. W. Bae, D. Yim, J. M. Park, J. Lee, H. S. Kim. Mechanical behavior and solid solution strengthening model for face centered cubic single crystalline and polycrystalline high-entropy alloys. Intermetallics 98 (2018) 89-94. DOI: https://doi.org/10.1016/j.intermet.2018.04.022

[16] Reshma Sonkusare, Aditya Swain, M. R. Rahul, Sumanta Samal, N. P. Gurao, Krishanu Biswas, Sudhanshu S.Singh, N. Nayan. Establishing processing-microstructure-property paradigm in complex concentrated equiatomic CoCuFeMnNi alloy. Materials Science and Engineering: A 759 (2019) 415-429. DOI: https://doi.org/10.1016/j.msea.2019.04.096

[17] Stephane Gorsse, Daniel B. Miracle, Oleg N. Senkov. Mapping the world of complex concentrated alloys. Acta Materialia 135 (2017) 177-187. DOI: https://doi.org/10.1016/j.actamat.2017.06.027

[18] M. Komarasamy, K. Alagarsamy, R. S. Mishra. Serration behavior and negative strain rate sensitivity of $\mathrm{Al}_{0.1} \mathrm{CoCrFeNi}$ high entropy alloy. Intermetallics 84 (2017) 20-24. DOI: https://doi.org/10.1016/j.intermet.2016.12.016

[19] S. R. Reddy, M. Z. Ahmed, G. D. Sathiraj, P. P. Bhattacharjee. Effect of strain path on microstructure and texture formation in cold-rolled and annealed FCC equiatomic CoCrFeMnNi high entropy alloy. Intermetallics $87 \quad$ (2017) 94-103. $\quad$ DOI: https://doi.org/10.1016/j.intermet.2017.04.016

[20] T. S. Reddy, I. S. Wani, T. Bhattacharjee, S. R. Reddy, R. Saha, P. P. Bhattacharjee. Severe plastic deformation driven nanostructure and phase evolution in a $\mathrm{Al}_{0.5} \mathrm{CoCrFeMnNi}$ dual phase high entropy alloy. Intermetallics $91 \quad$ (2017) 150-157. $\quad$ DOI: https://doi.org/10.1016/j.intermet.2017.09.002

[21] R. Sriharitha, B. S. Murty, R. S. Kottada. Phase formation in mechanically alloyed AlxCoCrCuFeNi (x= 0.45, 1, 2.5, 5 mol) high entropy alloys. Intermetallics 32 (2013) 119-126. DOI: https://doi.org/10.1016/j.intermet.2012.08.015

[22] Peitang Wei, Cheng Lu, Kiet Tieu, Lihong Su, Guanyu Deng, Wenbin Huang. A study on the texture evolution mechanism of nickel single crystal deformed by high pressure torsion. $\begin{array}{llllllll}\text { Materials Science } \& \quad \text { Engineering A } 684 & \text { (2017) 239-248. DOI: }\end{array}$ https://dx.doi.org/10.1016/j.msea.2016.11.098

[23] Abdel Yazid Khereddine, Fayçal Hadj Larbi, Hiba Azzeddine, Thierry Baudin, François Brisset, Anne-Laure Helbert, Marie-Hélène Mathon, Megumi Kawasaki, Djamel Bradai, Terence G. Langdon. Microstructures and textures of a $\mathrm{Cu}-\mathrm{Ni}-\mathrm{Si}$ alloy processed by high-pressure torsion. Journal of Alloys and Compounds 574 (2013) 361-367. DOI: https://doi.org/10.1016/j.jallcom.2013.05.051

[24] L.S. Toth, C.F. Gu, B. Beausir, J.J. Fundenberger, M. Hoffman. Geometrically necessary dislocations favor the Taylor uniform deformation mode in ultra-fine-grained polycrystals. Acta Materialia 117 (2016) 35-42. DOI: http://dx.doi.org/10.1016/j.actamat.2016.06.062

[25] Wei Chen, Lin Xiao, Qiaoyan Sun, Jun Sun. Effect of the initial grain size on grain refinement in Ti-2Al-2.5Zr alloy subjected to multi-impact process. Materials Science and Engineering A 554 (2012) 86-94. DOI: https://doi.org/10.1016/j.msea.2012.06.019

[26] L. S. Toth, P. Gilormini and J. J. Jonas. Effect of rate sensitivity on the stability of torsion textures. Acta Metallurgica 36 (1988) 3077-3091. DOI: https://doi.org/10.1016/00016160(88)90045-4 
[27] L. S. Toth, K. W. Neals and J. J. Jonas. Stress response and persistence characteristics of the ideal orientations of shear textures. Acta Metallurgica 37 (1989) 2197-2210. DOI: https://doi.org/10.1016/0001-6160(89)90145-4

[28] Tazuddin, N.P. Gurao, Krishanu Biswas. In the quest of single phase multi-component multiprincipal high entropy alloys. Journal of Alloys and Compounds 697 (2017) 434-442. https://doi.org/10.1016/j.jallcom.2016.11.383

[29] Tazuddin, Krishanu Biswas, N.P. Gurao. Deciphering micro-mechanisms of plastic deformation in a novel single phase fcc-based $\mathrm{MnFeCoNiCu}$ high entropy alloy using crystallographic texture. Materials Science \& Engineering A 657 (2016) 224-233. https://doi.org/10.1016/j.msea.2016.01.065

[30] Reshma Sonkusare, P. Divya Janani, N.P. Gurao, S. Sarkar, S. Sen, K.G. Pradeep, Krishanu Biswas. Phase equilibria in equiatomic CoCuFeMnNi high entropy alloy. Materials Chemistry and Physics 210 (2018) 269-278. DOI: https://doi.org/10.1016/j.matchemphys.2017.08.051

[31] Rani Agarwal, Reshma Sonkusare, Saumya R. Jha, N.P. Gurao, Krishanu Biswas, Niraj Nayan. Understanding the deformation behavior of CoCuFeMnNi high entropy alloy by investigating mechanical properties of binary ternary and quaternary subsets. Materials and Design 157 (2018) 539-550. DOI: https://doi.org/10.1016/j.matdes.2018.07.046

[32] S. Mohanty, T. N. Maity, S. Mukhopadhyay, S. Sarkar, N. P. Gurao, S. Bhowmick, Krishanu Biswas. Powder metallurgical processing of equiatomic AlCoCrFeNi high entropy alloy: Microstructure and mechanical properties. Materials Science and Engineering: A 679 (2017) 299-313. DOI: https://doi.org/10.1016/j.msea.2016.09.062

[33] R. Lebensohn, C. Tomé. A self-consistent anisotropic approach for the simulation of plastic deformation and texture development of polycrystals: Application to zirconium alloys. Acta Metallurgica 41 (1993) 2611-2624. DOI: https://doi.org/10.1016/0956-7151(93)90130-K

[34] C. Tomé, G.R. Canova, U.F. Kocks, N. Christodoulou, J.J. Jonas. The relation between macroscopic and microscopic strain hardening in FCC polycrystals. Acta Metallurgica 32 (1984) 1637-1653. DOI: https://doi.org/10.1016/0001-6160(84)90222-0

[35] La'szlo' S. To'th, Yuri Estrin, Rimma Lapovok, Chengfan Gu. A model of grain fragmentation based on lattice curvature. Acta Materialia 58 (2010) 1782-1794. DOI: https://doi.org/10.1016/j.actamat.2009.11.020

[36] Saiyi Li, Irene J. Beyerlein, Mark A.M. Bourke. Texture formation during equal channel angular extrusion of fcc and bcc materials: comparison with simple shear. Materials Science and Engineering A 394 (2005) 66-77. DOI: https://doi.org/10.1016/j.msea.2004.11.032

[37] K. G. Pradeep, G. Herzer, P. Choi, D. Raabe. Atom probe tomography study of ultrahigh nanocrystallization rates in $\mathrm{FeSiNbBCu}$ soft magnetic amorphous alloys on rapid annealing. Acta Materialia 68 (2014) 295-309. DOI: https://doi.org/10.1016/j.actamat.2014.01.031

[38] N. Mattern, J. H. Han, K. G. Pradeep, K. C. Kim, E. M. Park, D. H. Kim, Y. Yokoyama, D. Raabe, J. Eckert. Structure of rapidly quenched $\left(\mathrm{Cu}_{0.5} \mathrm{Zr}_{0.5}\right)_{100-x} \mathrm{Ag}_{x}$ alloys $(x=0-40$ at.\%). 
\begin{tabular}{lllllll}
\hline Journal of Alloys and Compounds 607 & (2014) 285-290. DOI:
\end{tabular} https://doi.org/10.1016/j.jallcom.2014.04.047

[39] F. Otto, A. Dlouhy, K. G. Pradeep, M. Kubenova, D. Raabe, G. Eggeler, E. P. George. Decomposition of the single-phase high-entropy alloy $\mathrm{CrMnFeCoNi}$ after prolonged anneals at intermediate temperatures. Acta Materialia 112 (2016) 40-52. DOI: https://doi.org/10.1016/j.actamat.2016.04.005

[40] J. G. Swadener, E. P. George, G. M. Pharr. The correlation of the indentation size effect measured with indenters of various shapes. Journal of Mechanics and Physics of Solids 50 (2002) 681-694. DOI: https://doi.org/10.1016/S0022-5096(01)00103-X

[41] K. Durst, B. Backes, M. Goken. Indentation size effect in metallic materials: Correcting for the size of the plastic zone. Scripta Materialia 52 (2005) 1093-1097. DOI: https://doi.org/10.1016/j.scriptamat.2005.02.009

[42] Anita Heczel, Megumi Kawasaki, Janos L. Labar, Jae-il Jang, Terence G. Langdon, Jeno Gubicza. Defect structure and hardness in nanocrystalline CoCrFeMnNi High-Entropy Alloy processed by High-Pressure Torsion. Journal of Alloys and Compounds 711 (2017) 143-154. DOI: https://doi.org/10.1016/j.jallcom.2017.03.352

[43] R.S. Ganji, P. S. Karthik, K. Bhanu Sankara Rao, K. V. Rajulapati. Strengthening mechanisms in equiatomic ultrafine grained $\mathrm{AlCoCrCuFeNi}$ high-entropy alloy studied by micro- and nanoindentation methods. Acta Materialia 125 (2017) 58-68. DOI: https://doi.org/10.1016/j.actamat.2016.11.046

[44] Soroosh Naghdy, Leo Kestens, Stijn Hertelé, Patricia Verleysen. Evolution of microstructure and texture in commercial pure aluminum subjected to high pressure torsion processing. Materials Characterization 120 (2016) 285-294. DOI: http://dx.doi.org/10.1016/j.matchar.2016.09.012

[45] H. Beladi, P. Cizek, P. D. Hodgson. On the characteristics of substructure development through dynamic recrystallization. Acta Materialia 58 (2010) 3541. DOI: https://doi.org/10.1016/j.actamat.2010.02.026

[46] K. Huang, R.E. Logé. A review of dynamic recrystallization phenomena in metallic materials. Materials and Design 111 (2016) 548-574. DOI: https://doi.org/10.1016/j.matdes.2016.09.012

[47] Werner Skrotzki, Nils Scheerbaum, Carl-Georg Oertel, Roxane Arruffat-Massion, Satyam Suwas, La'szlo' S.To'th. Microstructure and texture gradient in copper deformed by equal channel angular pressing. Acta Materialia 55 (2007) 2013-2024. DOI: https://doi.org/10.1016/j.actamat.2006.11.005

[48] N.P. Gurao, R. Kapoor, Satyam Suwas. Effect of strain rate on evolution of the deformation microstructure and texture in polycrystalline copper and nickel. Metallurgical and Materials Transactions A 41 (2010) 2794-2804. DOI: 10.1007/s11661-010-0360-X 
[49] H. Beladi, P. Cizek, P. D. Hodgson. Texture and substructure characteristics of dynamic recrystallization in a Ni-30\%Fe austenitic model alloy. Scripta Materialia 61 (2009) 528-531. DOI: https://doi.org/10.1016/j.scriptamat.2009.05.013

[50] Sumeet Mishra, Manasij Yadava, K. Kulkarni, N.P. Gurao. A modified Taylor model for predicting yield strength anisotropy in age hardenable aluminium alloys. Materials Science \& Engineering A 699 (2017) 217-228. DOI: https://doi.org/10.1016/j.msea.2017.05.062

[51] C.H. Ca'ceresa and P. Luka'c. Strain hardening behavior and the Taylor factor of pure $\begin{array}{lllll}\text { magnesium. Philosophical Magazine } 88 \text { (2008) 977-989. DOI: } & \end{array}$ https://doi.org/10.1080/14786430801968611

[52] L. S. Toth and J. J. Jonas. Analytic Prediction of Texture and Length Changes During FreeEnd Torsion. Textures and Microstructures 10 (1989) 195-209. DOI: http://dx.doi.org/10.1155/TSM.10.195

[53] L. S. Toth, K. W. Neals and J. J. Jonas. Stress response and persistence characteristics of the ideal orientations of shear textures. Acta Metallurgica 37 (1989) 2197-2210. DOI: https://doi.org/10.1016/0001-6160(89)90145-4

[54] Hossein Beladi, Pavel Cizek, Peter D. Hodgson. Dynamic recrystallization of austenite in Ni-30 Pct Fe model alloy: microstructure and texture evolution. Metallurgical and Materials Transactions A40 (2009) 1175-1189. DOI: 10.1007/s11661-009-9799-Z

[55] L. S. Toth, C. F. Gu, B. Beausir, J. J. Fundenberger, M. Hoffman. Geometrically necessary dislocations favor the Taylor uniform deformation mode in ultra-fine-grained polycrystals. Acta Materialia 117 (2016) 35-42. DOI: https://doi.org/10.1016/j.actamat.2016.06.062

[56] J. Baczynski and J. J. Jonas. Texture development during the torsion testing of $\alpha$-iron and two IF steels. Acta Materialia 44 (1996) 4273-4288. DOI: https://doi.org/10.1016/1359$\underline{6454(96) 00114-0}$

[57] D. A. Hughes, R. Lebensohn, H. R. Wenk, A. Kumar. Stacking fault energy and microstructure effects on torsion texture evolution. Proc. R. Soc. London A 456 (2000) 921. DOI: http://rspa.royalsocietypublishing.org/

[58] Anita Heczel, Megumi Kawasaki, Janos L. Labar, Jae-il Jang, Terence G. Langdon, Jeno Gubicza. Defect structure and hardness in nanocrystalline CoCrFeMnNi High-Entropy Alloy processed by High-Pressure Torsion. Journal of Alloys and Compounds 711 (2017) 143-154. DOI: https://doi.org/10.1016/j.jallcom.2017.03.352

[59] Ramya Sree Ganji, P. Sai Karthik, K. Bhanu Sankara Rao, Koteswararao V. Rajulapati. Strengthening mechanisms in equiatomic ultrafine grained $\mathrm{AlCoCrCuFeNi}$ high-entropy alloy studied by micro- and nanoindentation methods. Acta Materialia 125 (2017) 58-68. DOI: https://doi.org/10.1016/j.actamat.2016.11.046 
[60] R. Sriharitha, B.S. Murty, Ravi S. Kottada. Alloying, thermal stability and strengthening in spark plasma sintered $\mathrm{Al}_{x} \mathrm{CoCrCuFeNi}$ high entropy alloys. Journal of Alloys and Compounds 583 (2014) 419-426. DOI: https://doi.org/10.1016/j.jallcom.2013.08.176

[61] Chaoyue Zhao, Xianhua Chen, Fusheng Pan, Jingfeng Wang, Shangyu Gao, Teng Tu, Chunquan Liu, Jiahao Yao, Andrej Atrens. Strain hardening of as-extruded Mg-xZn (x = 1,2,3 and 4wt\%) alloys. Journal of Materials Science \& Technology 35 (2019) 142-150. DOI: https://doi.org/10.1016/j.jmst.2018.09.015

[62] H. Mughrabi. The $\alpha$-factor in the Taylor flow-stress law in monotonic, cyclic and quasistationary deformations: Dependence on slip mode, dislocation arrangement and density. Current Opinion in Solid State and Materials Science 20 (2016) 411-420. DOI: https://doi.org/10.1016/j.cossms.2016.07.001

[63] Tianlin Huang, Linfei Shuai, Aneela Wakeel, Guilin Wu, Niels Hansen, Xiaoxu Huang. Strengthening Mechanisms and Hall-Petch Stress of ultrafine grained Al-0.3\% Cu. Acta Materialia 156 (2018) 369-378. DOI: https://doi.org/10.1016/j.actamat.2018.07.006

[64] Y. H. Wang, J. M. Kang, Y. Peng, T. S. Wang, N. Hansen, X. Huang. Hall-Petch strengthening in Fe-34.5Mn-0.04C steel cold-rolled, partially recrystallized and fully $\begin{array}{llllll}\text { recrystallized. } & \text { Scripta } & \text { Materialia } & 155 & \text { (2018) } & \text { 41-45. }\end{array}$ https://doi.org/10.1016/j.scriptamat.2018.06.019

[65] S. Esmaeili, D. J. Lloyd, W. J. Poole. Modeling of precipitation hardening for the naturally aged Al-Mg-Si-Cu alloy AA6111. Acta Materialia 51 (2003) 3467-3481. DOI: http://dx.doi.org/10.1016\%2FS1359-6454(03)00167-8

[66] Reshma Sonkusare. MTech thesis (2018) IIT Kanpur, India. Thermodynamic calculations and phase equilibria of equiatomic CoCuFeMnNi high entropy alloy.

[67] B. Straumal, V. Pontikis, A. Kilmametov, A. Mazilkin, S. Dobatkin, B. Baretzky. Competition between precipitation and dissolution in $\mathrm{Cu}-\mathrm{Ag}$ alloys under high pressure torsion. Acta Materialia 122 (2017) 60-71. DOI: https://doi.org/10.1016/j.actamat.2016.09.024

[68] B. Straumal, A. Kilmametov, G. Lopez, I. Lopez-Ferreno, M. No, J. San Juan, H. Hahn, B. Baretzky. High-pressure torsion driven phase transformations in $\mathrm{Cu}-\mathrm{Al}-\mathrm{Ni}$ shape memory alloys. Acta Materialia 125 (2017) 274-285. DOI: 10.1016/j.actamat.2016.12.003

[69] Kaveh Edalati, Yuki Hashiguchi, Pedro Henrique, R. Pereira, Zenji Horita, Terence G. Langdon. Effect of temperature rise on microstructural evolution during high-pressure torsion. Materials Science and Engineering: A 714 (2018) 167-171. DOI: https://doi.org/10.1016/j.msea.2017.12.095 